\title{
La mediación editorial de la literatura latinoamericana en Francia, 1945-2000
}

Un modelo de datos para el análisis y la visualización de redes

\section{Visualización de redes sociales del pasado: historia cultural, producción simbólica y datos}

El concepto de mediación editorial ubicado al centro del proyecto Médiation éditoriale, diffusion et traduction de la littérature latino-américaine en France, de 1945 à 2000 (MEDET LAT) permite un primer acercamiento al omnipresente término "red" como metáfora útil para empezar a comprender lo que sucede en el campo de la traducción de literaturas extranjeras en un país o región lingüística dados. Efectivamente, la mediación, y en particular la mediación que se produce en el dominio de la edición de literaturas es, por definición, un poner en contacto dos o más actores a través de ciertos códigos (traducción, diseño gráfico, paratextos editoriales) $\mathrm{y}$, por lo tanto, se puede entender como una red o una serie de redes que posibilitan el trabajo de presentar y hacer circular las literaturas en lenguas extranjeras en el contexto de un público nacional. Analizar y visualizar tales estructuras reticulares es entonces un momento clave de la investigación del fenómeno de la mediación de la literatura latinoamericana en Francia durante la segunda mitad del siglo XX, entendida ésta como un espacio documental que encarna un campo cultural y sociopolítico específico.

Modelizar la mediación editorial de la literatura latinoamericana en Francia como una serie de redes pone en juego la especificidad del campo cultural francés a lo largo de más de medio siglo. En 1945 Roger Caillois crea en la editorial Gallimard la colección La Croix du Sud e inaugura el periodo histórico que concierne al proyecto MEDET LAT. Al fundar esta colección de literatura latinoamericana en traducción, Caillois se convierte en un actor central de la mediación editorial francesa. Como señala Gustavo Guerrero, el gesto de crear una colección dedicada exclusivamente a la traducción de la literatura latinoamericana, teniendo en cuenta que ya Gallimard tenía desde 1931 la colección Du monde entier que publicaba literaturas en traducción, constituye un ambicioso proyecto editorial que acompaña el desarrollo y establecimiento del latino-americanismo francés como un campo disciplinario que buscaba establecer un discurso sobre América

Roberto Parejas, CY Cergy Paris Université

Ә Open Access. (c) 2022 Roberto Parejas, published by De Gruyter. (c)) BY-NC-ND This work is licensed under the Creative Commons Attribution-NonCommercial-NoDerivatives 4.0 International License.

https://doi.org/10.1515/9783110707557-012 
Latina que no pasara por el tamiz anglo-americano (Guerrero 2018, 200-204). Es en este contexto que tiene sentido hablar de redes de la mediación editorial en Francia. En efecto, y como Guerrero certeramente apunta, el surgimiento del latino-americanismo francés requirió la construcción de un dispositivo que agrupaba diversos organismos, disciplinas y prácticas, y en los que Caillois y Gallimard, y muchos otros actores del mundo editorial, cultural y político francés, participaban activamente (Guerrero 2018, 202). Los actores (instituciones, individuos y objetos materiales y simbólicos) que entran en contacto e interactúan lo hacen entonces en contextos organizacionales que promueven esas interacciones y esos contactos. Si bien la mediación editorial no se puede concebir como una única organización bien definida y estructurada (como es el caso de organizaciones gubernamentales, internacionales u ONGs), ya que la componen diversos organismos e individuos con visiones a veces discordantes, lo cierto es que, más allá de sus diferencias, las estrategias de las casas editoriales y sus actores (desde los autores mismos y los traductores hasta los editores y personas influyentes en este campo) que se abocaron a publicar la literatura latinoamericana en traducción seguían una pauta marcada por un trasfondo institucional mayor ejemplificado, ya sea por el latinoamericanismo francés, o de manera más general, por las políticas de estado hacia América Latina o las ideologías de grupos políticos. Todo ello debe ser puesto en una perspectiva histórica que acentúe los cambios de época dentro del periodo definido para este estudio.

Una historia cultural de la mediación editorial francesa es en gran parte el estudio histórico de este conjunto de organismos, individuos y productos culturales en sus intrincadas relaciones tanto en el contexto francés como en el ámbito global y en su dinámica histórica. El análisis y la visualización de las redes que forman la mediación editorial son un momento esencial en la investigación, una herramienta que sirve varios propósitos: explorar la forma general de la red o de las redes menores centradas en un actor, o recabar información cualitativa y "morfológica" sobre las estructuras organizacionales subyacentes a las actividades de los individuos, además de producir indicadores cuantificados sobre los agenciamientos de las relaciones (Grandjean 2017).

En su estudio de la Comisión Internacional de Cooperación Intelectual (CICI) de la Sociedad de las Naciones (SDN) entre 1919 y 1927 Martin Grandjean propone un "análisis multinivel complejo" en el que la naturaleza, el contenido y el proceso de explotación de las fuentes documentales determinan los modelos de redes y la formalización a emplearse (Grandjean 2017). Es decir, no se trata una aplicación abstracta de modelos sobre un corpus ya formalizado de antemano por procesos automáticos. Desde esta perspectiva, el trabajo sobre las fuentes, sobre su materialidad documental y desde la lógica de la huella archivística, adquiere una relevancia fundamental y es, como ha indicado Anne Baillot, la forma 
en que las humanidades digitales siguen siendo humanidades en todo el sentido de la palabra (Baillot 2018). Cada proyecto es diferente, pero ya se trate de una edición digital de un corpus textual para explorarlo a partir de preguntas específicas o una investigación a partir de archivos originalmente digitales o de metadatos recuperables digitalmente, hay dos aspectos que deben ser explicitados: "la définition du corpus-source et le type de modélisation qu'il s'agit d'y appliquer, qui est la grille de lecture numérique de l'interprétation" (Baillot 2018, para 3).

El proyecto digital MEDET LAT trabaja sobre sus fuentes para alcanzar varios objetivos, entre ellos un catálogo/archivo digital de metadatos y una estrategia de análisis y visualización de redes usando esos metadatos, y que serán explicitados en la segunda parte de este texto. Por el momento recordemos que las visualizaciones de redes en las Humanidades suelen tener como objetivo poner en relación una determinada producción (artística, científica) y una serie de actores (autores, editores, traductores). Como señala Baillot, las posibilidades de esta herramienta son amplias ya que puede permitir a la investigación humanística pasar de la dimensión micro a la macro y viceversa con facilidad, ofreciendo la promesa de representar la "totalidad" (siempre relativa y abierta) de las relaciones en un dominio dado (Baillot 2015). Por ejemplo, en el caso del estudio histórico de organizaciones, el análisis y visualización de redes suele cumplir esta promesa bajo ciertas condiciones. Grandjean ofrece una cuidadosa revisión de la metodología de análisis y visualización de redes en este campo de la disciplina histórica con ejemplos de su trabajo ya mencionado sobre la CICI y llega a la conclusión que, a pesar de los problemas que implica la traducción de los conceptos de la teoría de grafos al análisis histórico (y por extensión al trabajo en las Humanidades en general), el análisis y la visualización de redes puede ofrecer ventajas al investigador si se cuenta con un corpus bien definido y ubicado en un "espacio documental” formalizado. Esta aproximación puede dar la impresión de que se trata de un corpus "total” y así llevar al investigador a interpretaciones con poco fundamento; sin embargo, si se mantiene la conciencia de que se trata de un corpus limitado y formalizado, es posible, a partir de esa base, enriquecer el análisis estructural de la red con una aproximación transversal. De este modo, nos dice Grandjean, las operaciones seriales sobre los documentos y la información estructural que se obtiene de éstas, se pueden cruzar con temáticas y problemáticas específicas del campo de estudio (Grandjean 2017). Así, el análisis y la visualización de redes complejas se pueden entender mejor como un proceso de exploración y menos como un resultado final y concluyente. La relación entre el análisis de las redes de la mediación editorial francesa y los estudios centrados en traductores o traducciones específicas, que constituyen uno de los trabajos fundamentales del seminario MEDET LAT, puede desarrollarse mucho más si 
se construye una aproximación sistemática y centrada en los metadatos bibliográficos básicos del proyecto y en otros datos que emergen de la investigación archivística y el análisis textual y sociohistórico.

Si bien la mediación editorial francesa de las literaturas extranjeras no es una organización formalmente delimitada como lo fue la CICI durante el periodo europeo de entreguerras y, a partir del fin de la Segunda Guerra Mundial, la UNESCO y, dentro de ésta, el proyecto de traducciones de la Colección de Obras Representativas de la Humanidad, la mediación editorial de las literaturas extranjeras en Francia puede ser pensada como un conjunto de instituciones, actores y políticas fuertemente ligados, muchas veces, por lazos de colaboración y, otras, confrontadas por la competencia comercial o la captura de prestigio y capital simbólico. El trabajo de Susanne Klengel en los archivos de la UNESCO en París sobre la Colección de Obras Representativas ha mostrado no solamente que ese proyecto editorial de alcance mundial fue un antecedente de los debates poscoloniales sobre el canon literario, sino que en esa discusión de mediados de siglo XX la literatura latinoamericana y los actores institucionales que la representaban (personal diplomático, muchos de los cuales eran intelectuales reconocidos en sus respectivos países y a nivel latinoamericano ) cumplieron un rol fundamental (Klengel 2018).

La UNESCO y su proyecto editorial universalista y "translatológico" (Klengel 2018) son parte orgánica de la mediación editorial francesa de la literatura latinoamericana. Las organizaciones, entre ellas las casas editoriales comerciales, pero también las universitarias o las de organizaciones internacionales, tienen estrategias que siguen lineamientos que vienen de políticas estatales y supranacionales, al mismo tiempo que los actores individuales (autores, traductores, editores), muchas veces con agendas muy personales, establecen sólidos vínculos de trabajo que atraviesan barreras institucionales y políticas. En conjunto, todas estas relaciones actualizan la visión que se tiene en determinado lugar y época sobre la función de las literaturas extranjeras en esa sociedad. El caso de Roger Caillois que dirige simultáneamente la colección La Croix du Sud de Gallimard y la serie Iberoamericana de la Colección de Obras Representativas de la UNESCO no es único dentro de la mediación editorial francesa. Guerrero señala que la participación del poeta mexicano Octavio Paz como editor de una antología de la poesía mexicana para la Serie Iberoamericana, cuya versión francesa, realizada por Guy Levis Mano, sería publicada en francés en 1952, a pesar de toda la polémica que rodeó su concepción y puesta en marcha, es testigo de la centralidad de la literatura latinoamericana en el proyecto translatológico de la UNESCO (Guerrero 2020). Esto sugiere una posible conexión entre este proyecto editorial internacional y las políticas culturales del estado francés respecto de América Latina, el surgimiento del latino-americanismo como disci- 
plina constituida en la academia gala y las estrategias de las casas editoriales en relación a las literaturas latinoamericanas. El análisis y visualización de estas redes podría poner en evidencia de una manera sistemática estas conexiones interinstitucionales e interpersonales, como los ya mencionados casos de Caillois o Paz, en base a un corpus de datos formalizados.

La mediación editorial como "totalidad abierta"1 se constituye en la interrelación de organizaciones (comerciales, culturales y políticas), de actores individuales y de objetos culturales que confluyen hacia un mismo objetivo, a saber: la traducción y difusión de la literatura latinoamericana en Francia. A pesar de que la mediación editorial carece de una única organización formal que aglutine jerárquicamente todas las funciones y determine una agenda común a todos los actores, esta actividad ha dejado una huella documental homogénea, formalizable a partir de conceptos y esquemas orientados a la computabilidad: los metadatos bibliográficos de las obras originales y de sus traducciones. Precisamente, la metodología de análisis y visualización de redes en los estudios históricos dependen, primero y esencialmente, de fuentes documentales en tanto conjunto de datos homogéneos, $y$, segundo, de los presupuestos del trabajo de investigación, los que implican una serie de decisiones y de selecciones más o menos arbitrarias (Grandjean 2017) que deben ser explicitadas y discutidas.

MEDET LAT pone al centro de su proyecto actual los metadatos bibliográficos de las traducciones y sus originales, pero es evidente que un estudio y relevo sistemático de los documentos de la UNESCO sobre la colección de Obras Representativas podría producir metadatos para analizar las redes constituidas al interior de la institución misma y sus conexiones con actores externos o que pasan por distintos contextos a lo largo del tiempo. Este conjunto de datos potenciales provenientes del espacio documental de la UNESCO es evidentemente complementario a los metadatos bibliográficos de MEDET LAT. Por ejemplo, es obvio que muchas publicaciones de la UNESCO forman parte de las redes de la mediación editorial francesa, como la ya mencionada Antologie de la poesie mexicaine, y que muchos actores individuales de esa institución funcionan como nodos que conectan redes de diferente tipo, así por ejemplo Roger Caillois, funge como mediador fundamental entre, por un lado, el proyecto editorial de

1 Tomo aquí libremente el concepto de "totalidad abierta" de Henri Lefebvre en Dialectical Materialism: "No expressions of dialectical materialism can be definite, but, instead of being incompatible and conflicting to each other, it may perhaps be possible for these expressions to be integrated into an open totality, perpetually in the process of being transcended, precisely in so far as they will be expressing the solutions to the problems facing concrete man" (Lefebvre 2009: 99). 
una organización internacional en el contexto de políticas culturales a gran escala y a nivel mundial, y, por el otro, la dinámica institucional de una serie de casas editoriales en un contexto nacional específico y dentro de los márgenes de un mercado editorial muy idiosincrático pero con alcance internacional. De igual manera, un enfoque en actores como Octavio Paz, que pasan por distintos contextos y funciones, desde puestos diplomáticos a funciones editoriales, amén de roles como autor y traductor, permite conectar las redes personales ego-centradas y el contexto global. Guerrero apunta, siguiendo a Froilán Inciso, que desde que ocupó un puesto en la Embajada de México en 1945, Paz tejió "an ample net of relations that connected different artistic, political and intellectual groups active in Post-War France" (Guerrero, anunciado en Bloomsbury.com n.d.) y, por tanto, podemos presuponer que muchas de esas conexiones se intersecan con la red de la mediación editorial francesa vista desde los metadatos bibliográficos. Otras quedan por fuera del campo puramente bibliográfico de la mediación editorial ya que refieren a relaciones personales y privadas dentro de un espacio biográfico, pero que, a su tiempo, pueden formar un conjunto de datos también formalizado que se puede integrar transversalmente a la red primaria. Podemos dar el ejemplo de la serie de entrevistas que el seminario MEDET LAT está conduciendo con traductores, editores y críticos y de algunas de las cuales ya se disponen transcripciones y archivos de video ('MEDET LAT - YouTube' n.d.) y que forman parte de un corpus textual actualmente en desarrollo.

\section{El proyecto digital MEDET LAT: modelo conceptual y conjunto de datos}

El punto de partida del proyecto digital MEDET LAT son, en un primer momento, los metadatos bibliográficos de obras literarias latinoamericanas traducidas al francés entre 1945 y 2000 (originales y traducciones) extraídos de los conjuntos de metadatos de la Bibliothèque nationale de France (BnF). Éstos forman el núcleo principal de la base de datos MEDET LAT cuyo modelo conceptual discutiremos brevemente en esta sección. El modelo prevé la inclusión, en un segundo momento, de una serie de conjuntos de datos, entre los cuales se pueden mencionar:

1. metadatos bibliográficos de la literatura secundaria que dan cuenta de las obras traducidas (crítica literaria aparecida en la prensa y en las revistas literarias y académicas en Francia); 
2. metadatos archivísticos de programas de radio en Francia en los que se entrevista a escritores, traductores, editores o críticos, se reseña las obras o se las representa adaptándolas al medio radial (repositorios del Institut national de l'audiovisuel INA $^{2}$ );

3. datos textuales (entidades nombradas y fechas) extraídos programáticamente ${ }^{3}$ de las entrevistas realizadas por el seminario MEDET LAT ${ }^{4}$ a traductores, editores y críticos;

4. datos textuales extraídos de paratextos editoriales seleccionados;

5. datos biográficos de los actores individuales (más allá de lugar y años de nacimiento y muerte) y datos institucionales de las organizaciones (más allá de la fecha de fundación) extraídos de los datos enlazados de la BnF;

6. datos provenientes de la investigación archivística:

a. cifras del tiraje de las obras traducidas que se pueden encontrar en repositorios como los Archives Nationales de France y Les archives administratives de la Bibliotèque nationale de France $(\mathrm{BnF})$;

b. metadatos de documentos en archivos de organizaciones internacionales como la UNESCO ${ }^{5}$;

2 La recepción francesa de la literatura latinoamericana en traducción puede estudiarse provechosamente a través del archivo radiofónico. Ver, como muestra, los metadatos de una entrevista de 1982 a Severo Sarduy en France Culture, disponible en línea a través del Catalogue du dépôt légal del Institut national de l'audiovisuel (INA) ('Severo-Sarduy' n.d.).

3 Para construir el corpus textual del proyecto, el equipo está empezando a explorar herramientas de anotación como Recogito ('Recogito' n.d.) o plataformas de desarrollo como eXist ('EXist-Db - The Open Source Native XML Database' n.d.).

4 "Le programme d'entretiens avec des traducteurs, avec des éditeurs et avec des critiques français de la littérature latino-américaine occupe une place de choix dans le projet MEDET LAT. Préparés à l'avance, par l'intermédiaire de plusieurs rencontres préalables entre l'interviewé et ses intervieweurs, les entretiens permettent d'analyser la carrière de différents médiateurs et de mieux comprendre les contextes historiques et le rôle qu'ils ont joué dans le processus de circulation des œuvres latino-américaines en France au cours de la seconde moitié du XXème siècle” ("Entretien avec Albert Bensoussan, réalisé par Gustavo Guerrero, par Ina Salazar et par Stephanie Decante” n.d.).

5 Ver, por ejemplo, una lista de obras a traducir en un documento de 1956, disponible en línea ("Programme de Traductions de l'UNESCO, A: Collection UNESCO d'oeuvres Représentatives UNESCO Bibliothèque Numérique” n.d.). Un documento similar fue anexado como apéndice en el artículo de Klengel ya citado (Klengel 2018, 148-55). 
c. datos y metadatos de documentos de las casas editoriales, por ejemplo, correspondencia y documentación administrativa, que se hallan en repositorios como el Institut Mémoires de l'édition contemporaine (IMEC) ${ }^{6}$ y en los archivos de las casas editoriales;

d. datos y metadatos de documentos que se hallan en archivos personales de traductores y editores, ya sea en colecciones privadas o en repositorios públicos como el mencionado IMEC;

e. premios concedidos a las traducciones

Los metadatos bibliográficos de las obras originales y las traducciones constituyen un corpus homogéneo en un espacio documental formalizado, es decir que el corpus está formado por un conjunto de entidades (libros publicados), seleccionadas de acuerdo a un criterio cronológico determinado y representadas de forma estandardizada por medio de esquemas de metadatos (por ejemplo, Dublin Core, MODS, etc.). Se trata de un espacio documental potencialmente muy grande que implica la totalidad de obras literarias traducidas del español latinoamericano y del portugués de Brasil al francés entre 1945 a 2000. El proyecto MEDET LAT ha decidido empezar con un corte del corpus que está determinado por una interpretación preliminar de la recepción de la literatura latinoamericana en Francia en traducción; es decir, una selección de autores y obras que cumplen ciertos criterios en cuanto a su recepción francesa, por ejemplo: cierta popularidad, recepción crítica, calidad de la traducción o premios literarios recibidos por la obra traducida. Esta selección preliminar de autores y obras se abre en una primera instancia para incluir a todas las obras traducidas de los autores preseleccionados y no solamente las que, por ejemplo, tuvieron mayor impacto crítico, y en una segunda instancia, a otros autores y obras no contemplados en la preselección. Este acercamiento iterativo y aproximativo tiene por función contrastar los presupuestos teórico-críticos sobre la recepción de la literatura latinoamericana en Francia con las redes que se producen en cada uno de estos casos, analizando y comparando su estructura y distribución a lo largo del tiempo. Es bueno recordar que el consenso crítico suele descansar en un sentido común no cuestionado. Las metodologías y herramientas digitales en

6 El IMEC es un repositorio fundamental para las investigaciones del grupo MEDET LAT. Contiene, por ejemplo, documentos internos de las casas editoriales, tales como los "dossier de fabrication" de los libros. Ver, por ejemplo, la referencia al dossier de Baiser de la femmearaignée de Manuel Puig en traducción de Albert Bensoussan ("Ressource 'Baiser de la femmearaignée / Puig, Manuel”" n.d.). 
Humanidades pueden ofrecer maneras creativas y rigurosas de revisar el consenso crítico sobre un autor, época o tema, tomando distancia de escala, lo que Franco Moretti llamó “distant reading” (Moretti 2013). Al respecto es interesante un reciente estudio, parte del proyecto Mapping the Republic of Letters, que, en base a metadatos y el análisis y la visualización de la red epistolar de Voltaire, cuestiona el consenso crítico que presuponía, fundamentándose en una sola obra de Voltaire (Lettres philosophiques), que el filósofo francés había sido marcado definitivamente por el pensamiento inglés hacia finales de los años 20 del siglo XVIII (Edelstein/Kassabova 2018; Edelstein 2016).

Los metadatos bibliográficos son entonces tanto un recurso que se puede usar para dar acceso a un conjunto de datos delimitados por una mirada teórica-metodológica, a través de una base de datos relacional y un interfaz de usuario, como una ventana hacia la reconstitución de las redes de la mediación editorial a partir de un conjunto de datos preparados especialmente para analizar y visualizar redes. Primero explicaré brevemente el modelo conceptual implementado para construir la base de datos y luego discutiré modelos de redes y los análisis y visualizaciones que permiten.

Las entidades centrales del modelo conceptual de la base de datos son “Translation” y “Original” (ver Figura 1). Ambas entidades representan, a través de metadatos seleccionados, las obras originales y sus traducciones al francés. De la gran cantidad de metadatos disponibles se han seleccionado sólo aquellos que son directamente útiles para la construcción de la base de datos y la producción de conjuntos de datos para el análisis y la visualización de redes. Para extraer los metadatos bibliográficos se han usado las Application Programming Interface (API) de la BnF (“ACCUEIL | Api.Bnf.Fr” n.d.), ya sea el servicio SRU (Search/Retrieve via URL) del "Catalogue général” para extraer los metadatos bibliográficos o el servicio SPARQL endpoint de data.bnf.fr para recuperar metadatos de autoridad (persona, organización, tema, obra, lugar) validados y enriquecidos por otros datos de la red semántica ("SPARQL Endpoint de Data.Bnf. Fr | Api.Bnf.Fr” n.d.). Como veremos más adelante, esta estrategia es fundamental para permitir una experiencia de utilización rica y fluida.

Por ejemplo, la tabla de la entidad “Translation” contiene los siguientes campos: 
Tabla 1: Entidad “Translation”.

\section{Translation}

\begin{tabular}{|c|c|}
\hline Campo & Descripción \\
\hline Id & $\begin{array}{l}\text { Número de identificación para cada entrada de la tabla, es decir } \\
\text { cada traducción }\end{array}$ \\
\hline BnF_Ark & $\begin{array}{l}\text { URI que refiere a la entrada de la obra en el catálogo electrónico de } \\
\text { la BnF }\end{array}$ \\
\hline Original_Id & $\begin{array}{l}\text { Número que identifica la obra original y que refiere a la tabla } \\
\text { "Original" }\end{array}$ \\
\hline Translation_Title & El título de la traducción \\
\hline Author_Id & $\begin{array}{l}\text { Número(s) que identifica(n) autor(es) y que refiere(n) a la tabla } \\
\text { "Person" }\end{array}$ \\
\hline Translator_Id & $\begin{array}{l}\text { Número(s) que identifican traductor(es) y que refiere(n) a la tabla } \\
\text { "Person" }\end{array}$ \\
\hline Collaborator_Id & $\begin{array}{l}\text { Número(s) que identifican a otro(s) colaborador(es) y que refiere(n) a } \\
\text { la tabla "Person" }\end{array}$ \\
\hline Publisher_Id & $\begin{array}{l}\text { Número(s) que identifica(n) la(s) casa(s) editorial(es) y que refiere(n) } \\
\text { a la tabla "Publisher" }\end{array}$ \\
\hline Collection_Id & $\begin{array}{l}\text { Número(s) que identifica(n) la(s) colección(es) y que refiere(n) a la } \\
\text { tabla "Collection" }\end{array}$ \\
\hline Place_Id & $\begin{array}{l}\text { Número que identifica el lugar de publicación y que refiere a la tabla } \\
\text { "Place" }\end{array}$ \\
\hline Date & Año de publicación \\
\hline Edition & Se indica, si se tiene constancia, la edición. \\
\hline Billingual? (Yes/No) & Indica si la publicación es una edición bilingüe o no \\
\hline Award_Id & $\begin{array}{l}\text { Si la traducción ha recibido un premio se indica el número de } \\
\text { identificación que refiere a la tabla "Award" }\end{array}$ \\
\hline Notes & $\begin{array}{l}\text { Cualquier otro aspecto relevante que no cabe en los anteriores } \\
\text { campos, por ejemplo, errores en los metadatos originales }\end{array}$ \\
\hline
\end{tabular}

Como se ve, la tabla "Translation" se alimenta de otras tablas creadas usando los metadatos bibliográficos extraídos. Así, todas las personas, sean autores, traductores, editores, ilustradores, etc., están alojadas en la tabla "Person" y de allí pasan a diversas tablas que usan esa información (ver Figuras 1 y 2). Eventualmente, a medida que la investigación de archivo progrese en ciertas áreas, se añadirán otras columnas, por ejemplo: número de ejemplares publicados.

Para limpiar, manipular y curar los datos y así poder crear las tablas de la base de datos y los conjuntos de datos para el análisis de redes, se usaron diversas herramientas, entre ellas, OpenRefine ("Openrefine.Github.Com” n.d.) y RStudio ("RStudio | Open Source \& Professional Software for Data Science Teams" n.d.). Para ilustrar esta parte del proceso de producción de los datos del proyecto, es interesante mostrar el paso desde los archivos Dublin Core (formato . 
XML) extraídos a través de la API BnF (ver Figura 3) a las tablas de datos relacionales (formato .CSV) pasando por la limpieza y manipulación de datos (ver Figuras 4 y 5). En esta fase del proceso, el investigador debía discernir los elementos y campos a mantenerse en las tablas curadas de aquellos elementos o campos que sirvieron para manipular los datos pero que no forman parte de las tablas ya curadas y listas para ingresar a un repositorio de datos para su conservación y acceso estandardizado. Por ejemplo, el elemento <dc:type > de Dublin Core que establece el tipo de recurso (texto impreso, imágenes, audiovisuales, multimedia, etc.) se mantuvo en las tablas en un principio para ayudar a filtrar ítems que no correspondían al tipo de recurso sobre el cual esta fase del proyecto se enfoca (texto impreso), pero luego se eliminó ya que en realidad no añadía un dato significativo en esta fase del proyecto. "Tipo de recurso" se convierte en un elemento significativo si se considera un corpus mixto de obras impresas y otro tipo de obras tales como las adaptaciones cinematográficas, radiofónicas, televisivas y teatrales de las obras traducidas. Como ya vimos, los metadatos de los programas radiofónicos relacionados a las obras traducidas serán integrados en una segunda fase del proyecto $\mathrm{y}$, por lo tanto, este campo en cuestión en realidad más que eliminarse, se guarda para su reutilización posterior.

Lo contrario pasa con el elemento <dc:description> de Dublin Core que, por lo general indica la colección de la casa editorial en la cual la obra ha sido publicada, ya que esta información es central para analizar la mediación editorial francesa. Este elemento indica también si se trata de una edición bilingüe, otro aspecto interesante para estudiar la mediación editorial. En ese caso, la limpieza y manipulación de los datos debe separar información que se halla en el mismo elemento de los metadatos, lo que ocurre también con <dc:publisher $>$ que contiene tanto el nombre de la editorial como el lugar de publicación, o de <dc:title $>$ que puede indicar el número de la edición y otras informaciones, además del título. En el ejemplo de la Figura 3 observamos que el elemento trae además el dato que la traducción al francés de esta obra de María Luisa Bombal desde la versión inglesa del original español, hecha por la misma Bombal.

Otro elemento Dublin Core utilizado por la BnF para codificar la información bibliográfica de su catálogo es <dc:contributor> que indica las personas (individuales o corporativas) que, además del autor o los autores, codificados como <dc:creator>, participan de alguna manera en la creación del recurso en cuestión. Traducteur es el ejemplo obvio para el proyecto MEDET LAT, pero $<$ dc:contributor $>$ incluye una serie amplia de roles, tomados de la taxonomía de la BnF, tales como Préfacier, Postfacier, Éditeur scientifique, Illustrateur o Auteur adapté, todos muy relevantes para la reconstrucción de las redes de la mediación editorial. 
Un aspecto central del trabajo con los datos en esta etapa es el enriquecimiento de los mismos a través de la adición de otros datos disponibles, como la extracción de ficheros de autoridad y sus identificadores para los nombres de persona y su inmediata incorporación a los conjuntos de datos, siendo el Virtual International Authority File ("VIAF" n.d.; "VIAF (Virtual International Authority File) | OCLC Developer Network” 2018) el estándar internacional. Esto se puede lograr de varias maneras, ya sea extrayendo directamente el identificador VIAF usando los servicios web de OCLC y herramientas ad-hoc, ya sea recuperando los ficheros de autoridad de la misma BnF a través de su servicio de datos ligados y web semántica (“Semanticweb_databnf_fr (Data.Bnf.Fr)” n.d.). Los ficheros de autoridad de la $\mathrm{BnF}$ se pueden reutilizar de diversas maneras usando herramientas de desarrollo como CubicWeb ("CubicWeb Semantic Web Framework” n.d.) que permiten la extracción e integración de datos de fuentes heterogéneas y en una variedad de formatos (CSV, MARC, Dublin Core, EAD-XML, $\mathrm{RDF}$ ) para fusionarlos, alinearlos y reagruparlos en una base de datos relacional SQL, o a través de ambientes de investigación como nodegoat que frecen la posibilidad de curar datos de investigación, generar análisis y visualizaciones y ligarlas a recursos de la red semántica (Bree and Kessels 2013a). De esta manera, los ficheros de autoridad se pueden reutilizar en la construcción de aplicativos para la web. En esta etapa del proyecto, el uso de este tipo de herramientas recién empieza a implementarse de forma general, ya que por el momento el enfoque está en el análisis de los metadatos bibliográficos para determinar el alcance del corpus del proyecto, la construcción de una base de datos relacional y un interfaz de búsqueda para explorar los datos de acuerdo a varios criterios y una serie de visualizaciones de las redes sociales que emergen de los datos.

\section{Base de datos, motor de búsqueda y criterios}

El proyecto MEDET LAT está en proceso de terminar la implementación del interfaz de búsqueda que será puesto a disposición para su uso por parte de un público especializado, entre académicos y profesionales de la edición, además del público general. Este prototipo será evaluado y posteriormente modificado o reemplazado por una versión mejorada. El prototipo de la base de datos y del motor de búsqueda está en su fase final de desarrollo. En lo que sigue presento ejemplos de búsquedas que ponen en juego las tablas Translation, Person y Collection y que muestran la capacidad de la base de datos y del buscador para 
presentar datos y conectar al usuario con las fuentes bibliográficas, es decir los registros originales del catálogo de la $\mathrm{BnF}$.

En estos ejemplos usamos un plugin para WordPress ${ }^{7}$ adaptándolo a las necesidades de los datos del proyecto con el objetivo de explorar y experimentar distintas maneras de dar acceso a los datos. En este caso solamente se trata de un filtro aplicado a una tabla, la versión final del prototipo del buscador conectará todas las tablas de la base de datos a partir de criterios de búsqueda (en forma de menú desplegable o conjunto de filtros) y que serán determinados en base a la experimentación en curso.

La tabla central de la base de datos es Translation. Es allí donde convergen la mayoría de los metadatos bibliográficos extraídos. En teoría, cualquier campo (es decir, cualquier columna de una tabla) puede ser indexado para ser fácilmente recuperado por un motor de búsqueda. Veamos un ejemplo de búsqueda donde ingresamos el nombre de un traductor destacado: Albert Bensoussan (ver Figura 6). Lo primero que notamos al ver los resultados de la búsqueda es que hay una inconsistencia en el nombre de pila del traductor en la primera entrada de los resultados: Robert en lugar de Albert. Afortunadamente, nuestro modelo de datos contempla el uso del identificador ARK (Archival Resource Key) en cada entrada de la tabla Translation y, a través del plugin podemos acceder a los otros campos de la tabla y cliquear en el identificador ARK para acceder al registro original del catálogo electrónico de la BnF (ver Figuras 7 y 8). De esta manera podemos deducir que el error provenía de los metadatos originales en el libro impreso y que se mantuvieron en los metadatos recuperados a partir de archivos Dublin Core. El registro MARC del catálogo de la BnF aclara el error al registrar el título (“Titre(s)"), pero lo mantiene en el campo “Autre(s) Auteur(s)". Esta manera de explorar los datos permite continuar con el proceso de curadoría de los datos a partir de su puesta en escena experimental en distintos tipos de buscadores y, además, ayuda a tomar decisiones que pueden afectar positivamente la experiencia del usuario. En este caso, se puede concluir que la mejor solución es corregir la entrada correspondiente en los datasets del proyecto y registrar el error en el campo "Notes" de la tabla "Translation".

7 Usar WordPress para construir la primera versión de la plataforma del proyecto determina las decisiones sobre la implementación del motor de búsqueda para la base de datos. Actualmente estamos experimentando con varios plugin ya existentes, como WP Data Access (Schulz n.d.), pero nuestro objetivo es llevar la plataforma a un ambiente y ecosistema digitales en el que podamos programar e implementar un buscador que agregue y organice datos heterogéneos provenientes de distintos proyectos y fuentes. 
Lo que notamos en esta lista de resultados es la variedad y cantidad de las traducciones de Bensoussan. Esta variedad contiene una multiplicidad de relaciones y de agenciamientos entre algunos de los actores implicados en la producción del objeto libro y en su circulación e integración en el discurso público. Un buscador debe ofrecer la posibilidad de acceder preliminarmente a esas relaciones a través de criterios de búsqueda que, utilizando los campos y términos definidos por el modelo conceptual implementado, pueden filtrar los datos para producir resultados útiles e interesantes para el usuario. El prototipo que está en desarrollo establecerá las relaciones entre traductor y casas editoriales a partir de la tabla "Person" y la tabla "Publisher" mediadas por la tabla "Translation", donde una búsqueda SQL (Structure Query Language) conectará un traductor específico y una casa editorial específica y producirá un resultado positivo (con lista de resultados), si existe una conexión, o negativo (con mensaje para el usuario), si no existe. El buscador debe además poder mostrar al usuario el acceso a recursos externos, por ejemplo, archivos de video en repositorios audiovisuales, particularmente las entrevistas a traductores, editores y críticos. Pensemos en la ya citada entrevista a Benssoussan: una búsqueda de "Bensoussan" por nombre de persona debe dar como uno de sus resultados los metadatos del recurso audiovisual de la tabla "Interview" que se hallan ligados a través de la tabla "Person", incluyendo el enlace al repositorio audiovisual que contiene el archivo de video.

Un motor de búsqueda equipado con una variedad de criterios es una manera eficiente de acceder a los datos de investigación y de empezar a estudiar ciertas relaciones en el corpus de metadatos del proyecto. La herramienta más adecuada para continuar esa exploración más allá de la base de datos es el análisis y visualización de redes, es decir, la puesta en relación de los actores de la mediación editorial a partir de los metadatos bibliográficos para mapear comunidades a lo largo de ejes espaciotemporales, localizar redes ego-centradas y entender la participación de los actores en las redes en base a indicadores estadísticos.

\section{Conjunto de datos y modelos de redes}

Es necesario notar que hay dos modelos fundamentales de redes, que se conocen por su terminología en inglés: one-mode y two-mode. El modelo de los metadatos bibliográficos, en una primera instancia, se concibe como two-mode, ya que siempre se trata de conectar conjuntos de entidades de distinto tipo, en nuestro caso, objetos culturales (textos manuscritos, libros impresos, documentos) y actores indi- 
viduales (autores, traductores o editores) o, incluso, actores colectivos (organizaciones e instituciones que aparecen como creadores de documentos y recursos). Sin embargo, y al igual que en el caso de las redes de correspondencia epistolar o de memorandos y documentos administrativos, el modelo two-mode se proyecta en un modelo one-mode en el que el objeto cultural es descentrado y se convierte en el vínculo entre actores, es decir entre entidades del mismo tipo, por ejemplo, las relaciones entre autor, traductor y editor a partir de su coparticipación en el mismo proyecto editorial. El análisis y visualización de redes puede navegar entre estos dos modos para representar la mediación editorial desde distintas perspectivas; por ejemplo, la red de la mediación editorial se puede mostrar desde una perspectiva two-mode si las obras se convierten en los actores centrales y la relación entre actores (sean individuos u organizaciones) pasa siempre por la mediación de la obra; tendremos también una dinámica similar si convertimos a la casa editorial en el actor central.

Otra posibilidad de actualizar el modelo one-mode es conectar a las obras originales y traducidas, lo que ofrece una ventana para observar la dinámica de los objetos culturales en relación a su procedencia geo-cultural en el contexto global de la red de mediación editorial, tal el caso de una red en la que las obras traducidas y las originales son los actores centrales y los metadatos lingüísticos y espacio-temporales (como características de los nodos de la red) permiten analizar y visualizar las variaciones de origen geográfico y lingüístico de la red a través del tiempo.

Ahora bien, como indica Grandjean, sólo se llega a un conjunto de datos homogéneos en un espacio documental formalizado a partir de "[a] fastidious retrieval of a selection of documents metadata" (Grandjean n.d.), es decir a través de un trabajo minucioso y detallado sobre las fuentes documentales, uno de los rasgos definitorios del trabajo humanístico desde sus orígenes y que es propio de la organización de la información y el conocimiento en todas las culturas desde la antigüedad. La creación de índices que conectan actores y objetos culturales, y luego a los actores entre sí, sólo es posible si el investigador aplica sobre los metadatos bibliográficos disponibles una perspectiva hermenéutica y crítica, y esto mucho antes de empezar a crear flujos de trabajo automatizados que permitan, por ejemplo, como ya vimos, la extracción programática de datos vía APIs y la subsiguiente conversión en masa de archivos informáticos a otros formatos más adecuados para la manipulación de datos.

Una vez los metadatos bibliográficos son transformados en conjuntos de datos en base a determinadas entidades (traducción, original, persona, rol de persona, casa editorial, colección, lugar, año, etc.), entonces se puede iniciar la creación de índices y matrices para analizar y visualizar redes. Pero antes incluso de crear índices y matrices con datos reales, es bueno hacer un ejercicio 
de modelización abstracta que nos ubique en el espacio isotrópico ${ }^{8}$ de las redes donde, en teoría, todo puede estar conectado con todo. Visualicemos primero una red (Figura 9) en la que podemos observar las relaciones entre las entidades Author X, Translator A, Work 1 (original) y Work 1' (traducción). Para visualizar esta red fue necesario crear, por una parte, una lista de nodos que, en este caso, es una lista mixta de entidades donde caben tanto las obras (original y traducción) como los actores básicos involucrados en el proceso (autor y traductor), además de una lista de arcos o ligaduras que conectan a los nodos entre sí, en este caso cada nodo está conectado con los otros nodos ya que: Author X ha creado una obra, ha participado en la traducción y, por lo tanto, ha colaborado con el traductor, al igual que Translator A ha traducido la obra original, produciendo un nuevo objeto (la traducción) y ha interactuado de diversas formas con el autor de la obra original. De igual modo, ambas versiones, Work 1 y Work 1', están ligadas por el hecho de que una es el origen de la otra. Es de notar que la relación de autor y traductor con la obra original y la traducción se concibe como unidireccional (lo cual se indica con una flecha de una dirección), mientras que la relación autor-traductor es bidireccional (indicado por una línea sin flechas) dado el carácter colaborativo, en principio, de esa relación; por otra parte, la relación entre original y traducción puede ser conceptualizada como bidireccional ya que, en la práctica, no vamos a visualizar todas estas relaciones al mismo tiempo, como ya adelantamos al explicar los modelos one-mode y two-mode.

Observemos otro modelo abstracto (Figura 10) en el que añadimos a Author $Z$ y Translator B, además de 3 originales y 3 traducciones. Esta visualización, al igual que la anterior fue creada con Gephi ("Gephi - The Open Graph Viz Platform" n.d.), la diferencia es que esta última ha sido tratada con el algoritmo ForceAtlas 2 para detectar comunidades y ayudar en la interpretación visual de los grafos (Jacomy et al. 2014). También podemos observar que se ha aplicado una gradación de color para señalar la distancia entre Author X y los otros nodos. De este modo podemos claramente distinguir como Translator B no solo colabora con Translator A en la traducción de Work 3 de Author X, sino que además ha traducido por su cuenta Work 2 para Author X y Work 4 para Author Z.

\footnotetext{
8 Franco Moretti define el espacio isotrópico de las modelizaciones geométricas como una "abstracción teórica" que presupone que el movimiento puede ocurrir hacia todas direcciones de la misma manera; el problema de Moretti es la representación del espacio de la narrativa y el movimiento o de los personajes, pero este desfase entre geometría y geografía social es similar a la diferencia que hay entre los modelos abstractos de redes y las redes sociales reconstruidas a partir de datos y metadatos concretos (Moretti 2007: 44, 56; nn. 4,12).
} 
Finalmente, podemos añadir a la lista de nodos la entidad Editor. En este grafo (Figura 11) se destaca el rol de Editor $\Sigma$ ocupando una posición intermediadora entre grupos, lo que apunta al hecho de que hay nodos que tienen menos conexiones pero que pueden cumplir una función de pasaje entre comunidades. Los actores categorizados como editores se determinan en base a los datos bibliográficos, por ejemplo, si se indica explícitamente el nombre del/a director/a de una colección editorial, como en el caso de Roger Callois y La Croix du Sud en Gallimard. O, alternativamente, si se conoce el nombre de la persona que dirige la colección, aunque no se indique explícitamente en los metadatos disponibles.

Baillot sugiere, en un contexto un poco diferente del de este proyecto, que

[L]'un des principaux avantages des visualisations de réseaux est de faire surgir visuellement quelque chose d'inattendu, de nouveau. En visualisant les données du réseau, on peut ainsi idéalement repérer les aspects d'un corpus qui ne sont pas visibles “à l'oeil nu", celui de la lecture humaine des mêmes textes. Cette démarche permet d'orienter la recherche vers les aspects qu'il s'agit ensuite de creuser de manière dite "qualitative”, par opposition au "quantitatif" utilisé pour réaliser des visualisations de données en nombre considérable. Avant d'avoir effectivement cette fonction heuristique, la visualisation suppose un certain nombre d'étapes préliminaires de travail au cours desquelles on teste d'abord les algorithmes sur des résultats connus pour vérifier qu'ils y mènent en effet. Ce n'est qu'après ce calibrage qu'on laisse la visualisation, à son tour, proposer des hypothèses (Baillot 2016, 88-89).

Estas consideraciones generales son válidas para cualquier proyecto que trabaje con conjuntos de datos provenientes de series formalizadas, ya se trate de documentos archivísticos de organizaciones, como en el caso del estudio de Grandjean sobre el CICI; correspondencia repertoriada, como el estudio de Baillot sobre la correspondencia de Karl Wilhelm Ferdinand Solger y el romanticismo berlinés (“Lettres et Textes: Le Berlin Intellectuel Des Années 1800” n.d.); o metadatos bibliográficos tomados de catálogos electrónicos, como en el caso de MEDET LAT.

\section{Interfaz de visualizaciones interactivas}

La siguiente fase del proyecto contempla el diseño e implementación de un interfaz para visualizaciones interactivas que se podría realizar usando herramientas ya disponibles para ello, por ejemplo el ya mencionado ambiente de investigación nodegoat (Bree and Kessels 2013a), o creando un aplicativo propio en colaboración con laboratorios especializados de las instituciones participantes en el proyecto. La alternativa de usar una herramienta como nodegoat es atractiva desde varios puntos de vista. Conceptualmente, nodegoat ofrece un marco en el cual proyectos de 
Humanidades Digitales que exploran redes complejas pueden analizar y visualizarlas a partir de un esquema basado en los presupuestos de la teoría del actor-red. Esta teoría conceptualiza personas, eventos, artefactos y fuentes de forma homogénea como objetos, en tanto que las jerarquías que emergen dependen únicamente de las relaciones que componen las redes ("Nodegoat | About" n.d.). A diferencia del marco conceptual que subyace a herramientas como Gephi, en nodegoat la diferencia entre modelos one-mode y two-mode pierde su relevancia crítica ya que todas las entidades son tratadas como objetos. Esta metodología ofrece flexibilidad al momento de pensar las relaciones y explorar sus trayectorias, permitiendo al investigador crear conjuntos de datos en base a modelos de datos propios, creados especialmente para el análisis y la visualización requeridos. El análisis relacional de los datos en nodegoat incluye contextualizaciones espaciales y cronológicas que permiten de forma inmediata el procesamiento, análisis y visualización de conjuntos de datos muy complejos. nodegoat también puede integrar y conectar datos ligados de la web semántica configurando SPARQL endpoints o recursos API para su uso consistente en, por ejemplo, el establecimiento de enlaces URI ("Nodegoat | About” n.d.), lo que permitiría, por ejemplo, el despliegue de la información bibliográfica y biográfica de los ficheros de autoridad de la BnF directamente desde las visualizaciones interactivas (ver Figuras 12, 13, y 14).

Finalmente, nodegoat es una herramienta que permite al investigador "traducir" preguntas de investigación a la teoría de redes a partir de conjuntos de datos y de la creación de modelos en los que las entidades son conceptualizadas desde una metodología object-oriented (Bree and Kessels 2013b). El usuario de este ambiente de investigación accede a crear modelos, curar datos y supervisar procesos. El marco conceptual para la creación de modelos de datos contempla la entidad Tipo poblada de Objetos, la entidad Clasificación con Categorías y la Clasificación en Reversa que define un filtro multifacetado. La estructura de un Tipo, por ejemplo, Translation, Person o Place, viene dada por los Objetos y Sub-Objetos y sus descripciones, donde Objeto puede alojar descripciones que son equivalentes a una columna/campo de una tabla/entidad en una base de datos relacional, mientras que el Sub-Objeto permite determinar los atributos contextualizados de un Objeto, por ejemplo, temporalidad o roles de actores (Figuras 15) y prepara el trabajo para el análisis de redes y las visualizaciones.

Una pregunta de investigación sobre la mediación editorial francesa de la literatura latinoamericana puede ser traducida a esta lógica. Por ejemplo, en nodegoat, el rol de mediadores como Roger Caillois puede ser descrito y evaluado a partir de los metadatos bibliográficos que producen cierta métrica estadística, pero ante todo porque puede ser localizado en una red extensa y densa, para la cual hay documentación contextual muy rica y variada. La colección La Croix du Sud de Gallimard, por ejemplo, es una red en sí misma. Explorar la red ego-centrada de 
Caillois podría hacer visible las relaciones de Caillois, La Croix du Sud y por extensión Gallimard, con otros proyectos editoriales, tal el caso del ya mencionado proyecto translatológico de la UNESCO y su Serie Iberoamericana. Caillois y escritores como el mexicano Octavio Paz no solamente están relacionados por lazos profesionales encasillados en funciones claras, como editor y autor, o traductor y autor, sino que coinciden en roles diplomáticos, administrativos o políticos, que son menos fijos y más fluidos, además de que muchas veces ejercen estos roles al mismo tiempo. En la Figura 16 vemos un sector de la red general donde el rol mediador de Caillois puede empezar a surgir más claramente, mientras que en las Figuras 17 y 18 podemos ver la presencia de Caillois en la red ego-centrada de Pablo Neruda como traductor de Alturas de Macchu Picchu, una sección de Canto general.

\section{Conclusiones}

¿Cómo ha cambiado la relación entre este tipo de actores entre 1945 y 2000? ¿Qué relaciones hay entre academia, organizaciones internacionales y casas editoriales en el contexto estudiado? ¿Cuál es la relevancia del latino-americanismo francés en este contexto? ¿Cómo participan las editoriales universitarias en la mediación editorial estudiada? Estos son ejemplos de preguntas que se pueden hacer usando los datos que el proyecto MEDET LAT ya tiene. La incorporación de datos nuevos que vendrían, por ejemplo, de un corpus textual que incluye entrevistas y biografías, enriquecería el conocimiento de nuestra red y aumentaría el alcance de los análisis y las visualizaciones al plantear, por ejemplo, preguntas sobre redes personales situadas en una escala cercana a la privacidad de individuos involucrados en la red. Un ejemplo central es la relación de Albert Bensoussan con los autores a quienes traducía y la descripción de su particular método de traducir, aspectos documentados en la entrevista de MEDET LAT como en otros textos. Armar los datos para analizar y visualizar la red global desde la red personal de Bensoussan usando los modelos de nodegoat es posible y, lo que es más importante, permitiría la escalabilidad del análisis y de las visualizaciones desde lo macro a lo micro $\mathrm{y}$ viceversa.

Hay muchos otros aspectos que discutir en el contexto del proyecto MEDET LAT en cuanto a la creación de conjuntos de datos, su curadoría y puesta en producción a través de análisis y visualizaciones. Para terminar, quiero mencionar la centralidad que van a tener metadatos como el rol de las personas involucradas en la red o la relación entre traducción y original para generar interesantes análisis a lo largo del tiempo y del espacio con la potencialidad de plantear nuevos contextos y problemas para la investigación. 


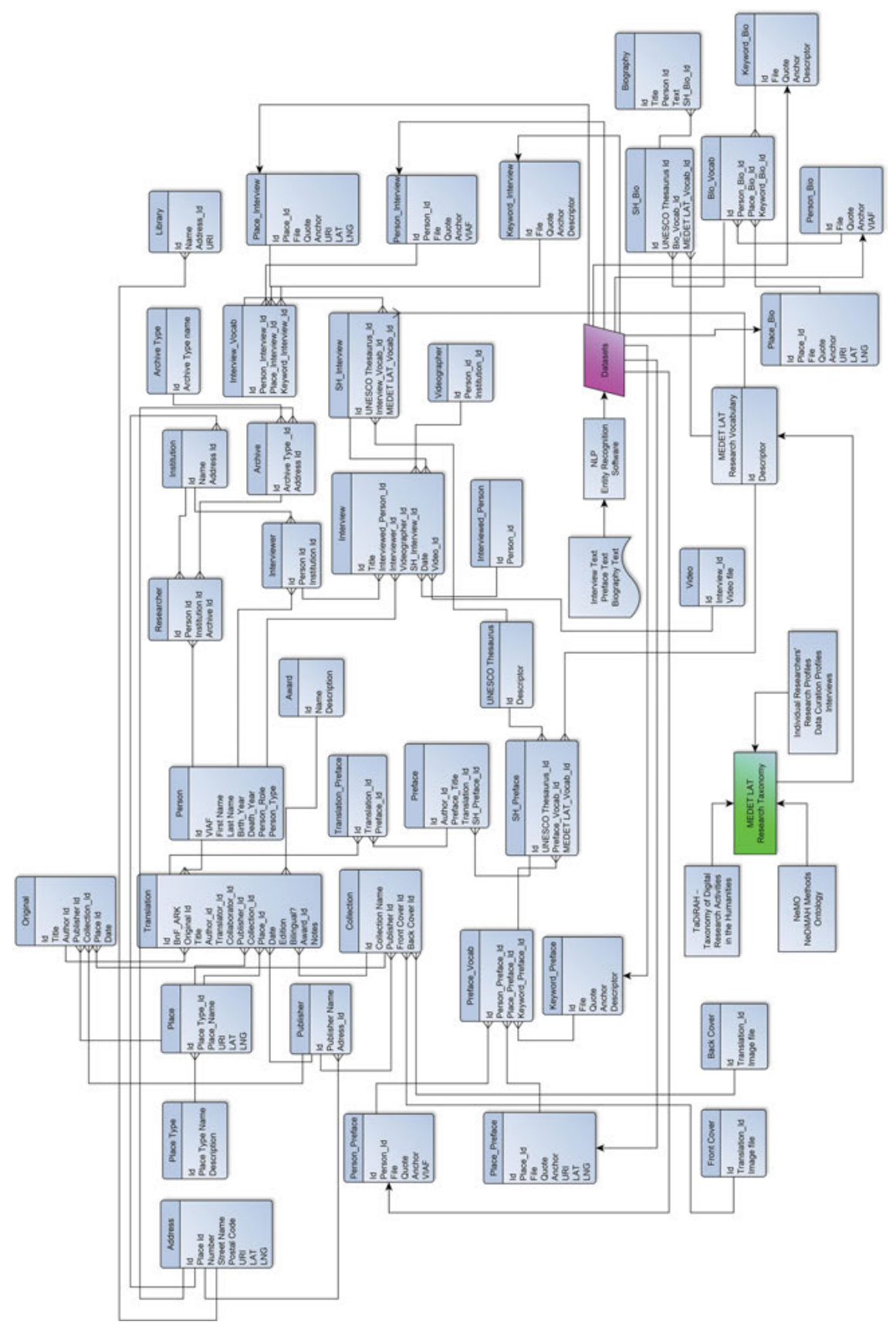

Figura 1 


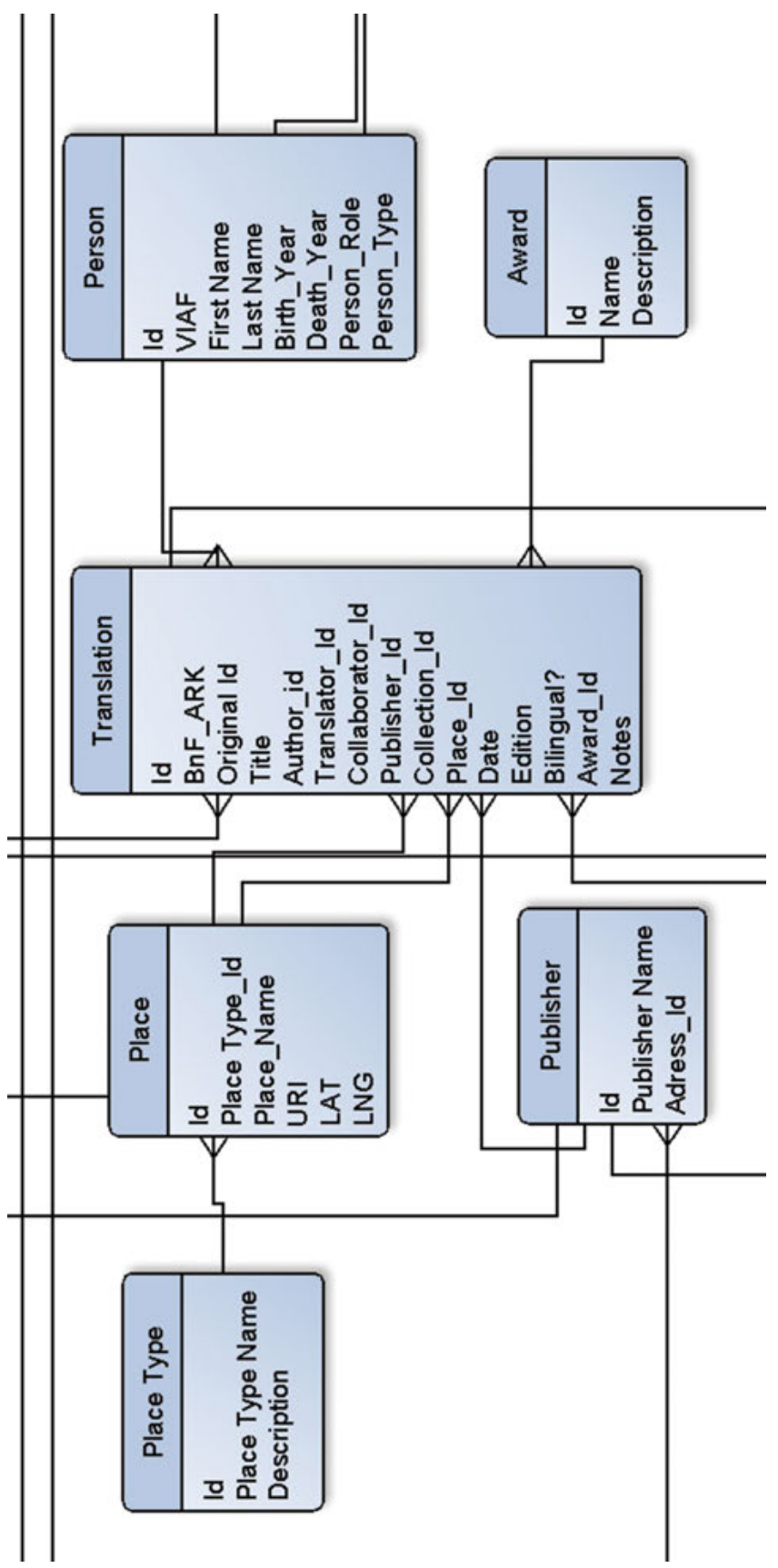

Figura 2 


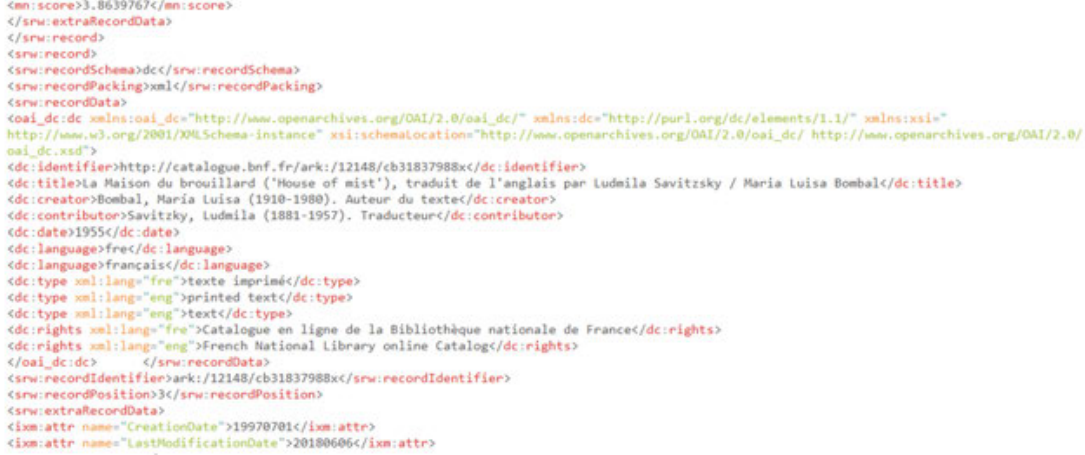

\section{Figura 3}

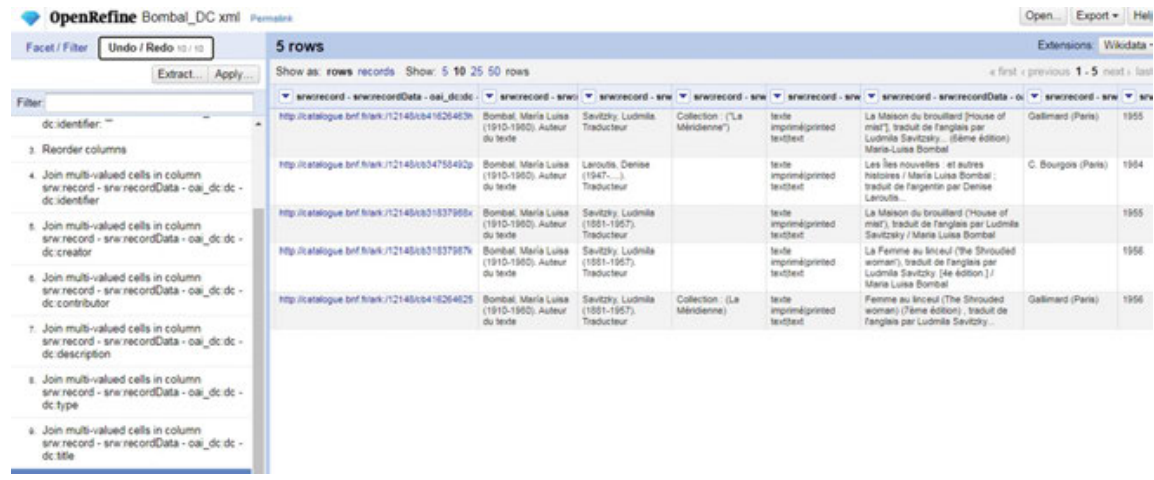

\section{Figura 4}

7i Translation_Database_final में to 0

File Edit View Insert Format Data Tools Add-ons Help Lasteditwas 3 minutesago

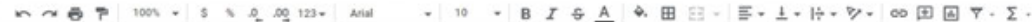

\begin{tabular}{|c|c|c|c|c|c|c|c|c|c|c|c|}
\hline \multicolumn{12}{|l|}{$f x$} \\
\hline & A & 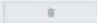 & c & o & E & F & o & H & 1 & 2 & $\mathrm{k}$ \\
\hline 101 & & Hitpilicatiogue & La Mason du broullard & Bombal, Mria Luisa & Savitasy, Ludmia & & & & 1955 & (Mouse of mist). & tradun de \\
\hline 102 & & Hito licatalogue. & Le partage des easx & Carpenter, Alepo & Drand, Rene L & & Gallimard & Pans & 1955 & (2e ed) & \\
\hline 103 & & Hitollicatiogue. & Cumboto & Diaz Sanchez, Ramon & Drand, Rent $4 \mathrm{C}$ Coryse & e. Andrite & Nouveles tadion f & Pais & 1955 & & \\
\hline 104 & & Hollisaralogue. & ILes Espaces chauds & Gerbasi, Vkente & calaton clavide & & & & 1255 & & \\
\hline 105 & & itiolichaloque & Chansons cubanes ef actres potmes & Gullen, Nikolas & Callon, Claude & & & & 1055 & & \\
\hline 100 & & Hitpilleatogue & Exgies artallaises & Gullen, Nicoliss & Caltion, Clasude & & & & 1965 & & \\
\hline 107 & & Hoilctaloque & IL Ourapan & Astunas, Mgue Angel & Pilemert Georges & & Galimard & Pans & 1965 & & \\
\hline 100 & & Hitplicatiogue & La Mason du broullard & Bombal, Meria Luisa & Savitzy, Ludmia & & Galimard & Pans & 1955 & House of mist tr: & traduit de $f$. \\
\hline 100 & & Hoflicatalogue. & Cumbato : roman. & Disz Sanchez Ramon & Durand, Rend $\mathrm{L}$ & & & & 1955 & & \\
\hline 110 & & Heollesalogue. & ILe Pape vert roman & Asturias, Mguel Anget & Momande, Francis de & & & & 1956 & & \\
\hline 111 & & Hellikarague. & Le Pape vert roman & Asturias, Maguel Angel & Momande, Francis de & & & & 1956 & & \\
\hline 172 & & Thenpilicatalogue & La Fenme au inced & Bombal, Mria Lusa & Savitzay, Ludmila & & & & 1956 & (the Strouded wo & voman), ter \\
\hline 113 & & Hellicanogue & Terres du sucre & Fregre, Gibeto & Orecchioni, Jean & & Gallimard & Pans & 1956 & & \\
\hline 114 & & inpellikatalogue: & Monument a la mer & Huidobro, Vicente & Verhesen, Fernand & & & & 1956 & & \\
\hline 115 & & thollicatiogue: & Dom Casmuto & Assis, Machado de | As & Momande, Francis de V & Version revur & Albin Michel & Paris & 1956 & & \\
\hline 118 & & holdillatiogue: & Joyage au point du jour & Mariano Picon Salas & Carysse, Jean| Canysuse, & 2. Andrite Du & Nourveles edoon ? & Paris & 1956 & & \\
\hline 113 & & 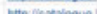 & & Daser fentions & 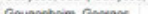 & & Makimus & Dowe & thise & & \\
\hline
\end{tabular}

\section{Figura 5}




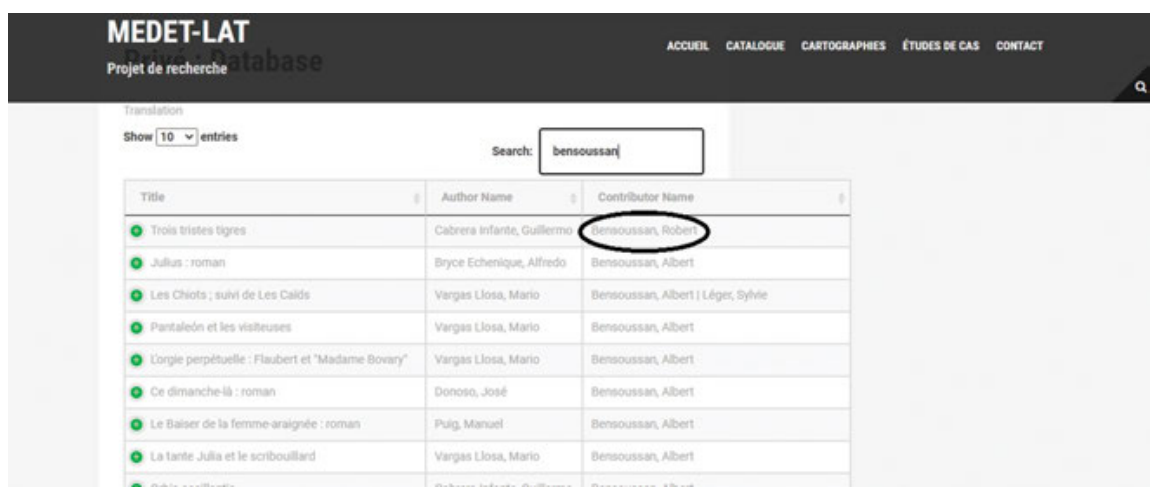

Figura 6

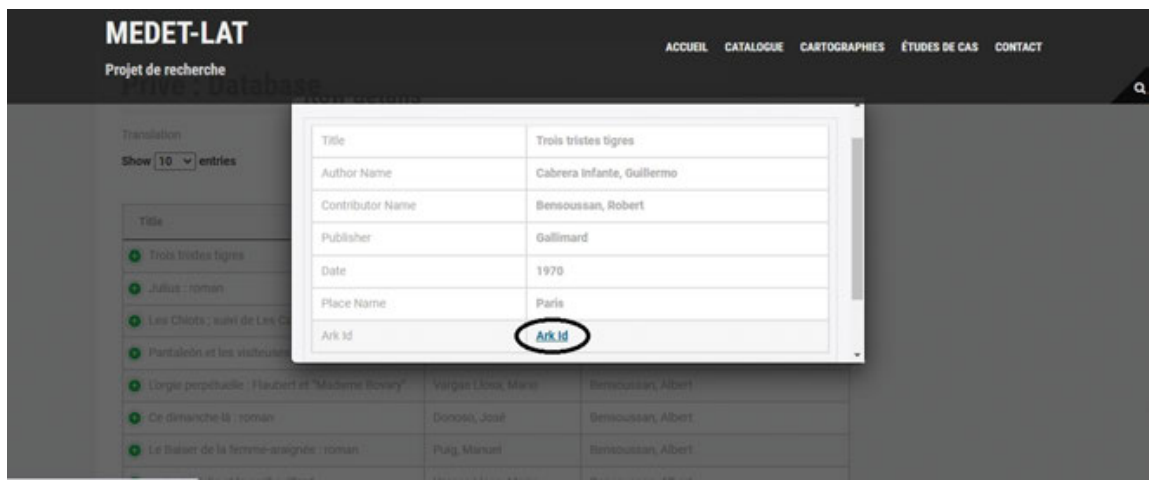

Figura 7 


\begin{tabular}{|c|c|c|c|c|c|c|c|c|}
\hline IBnF & Catalogu & génér & & & & is Esposenerenon & ned IV Note unequetson & Hesorique A \\
\hline & (1) Tout & $\checkmark a$ & 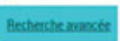 & Auteurs $A Z$ & SWJETSAZZ & Peradonouts core & 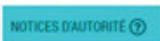 & DANS LANERS $\checkmark$ \\
\hline
\end{tabular}

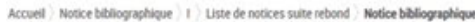

Notice bibliographique

\section{Notoce Au formumpoblic $\checkmark$}

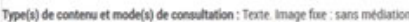

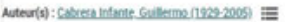

Thre conventionnel : Thes trites biges/tuncabl :

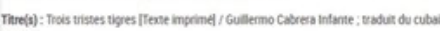

Pastevit

Trabuction \&e: : Tres tristes tigres

Patlication : I Paris: : Gallemard, 1970

Description mutitiele : 467 p : ill : $21 \mathrm{~cm}$

Note(t) : Du monde enoes

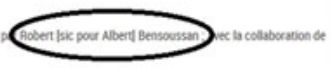

Sert lisic pour Mbert Bensoussan se la collaboration de

\section{Figura 8}

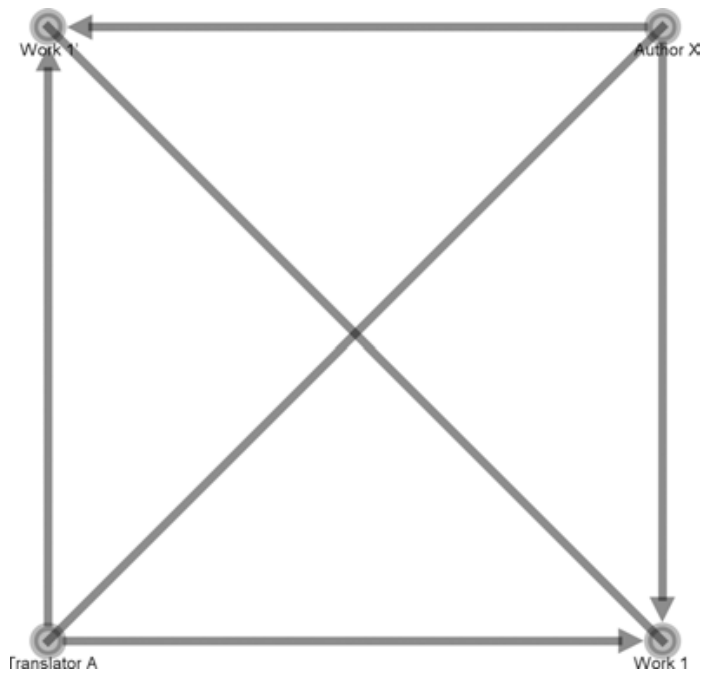

Figura 9

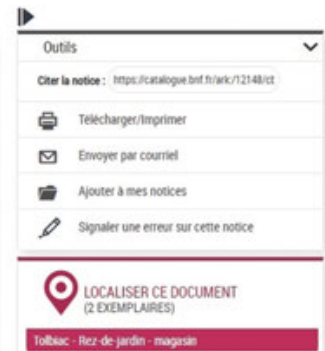

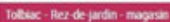




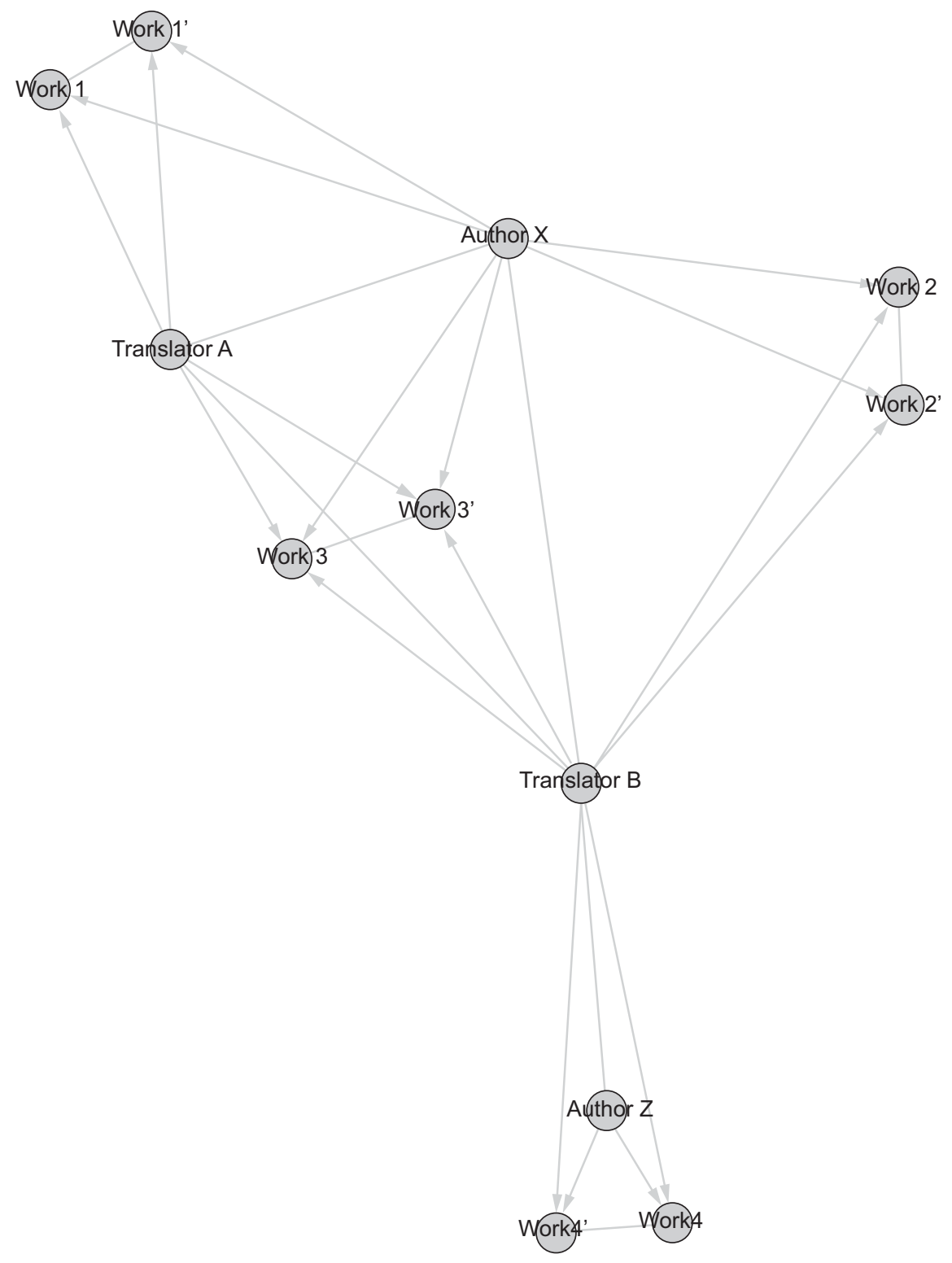

Figura 10 


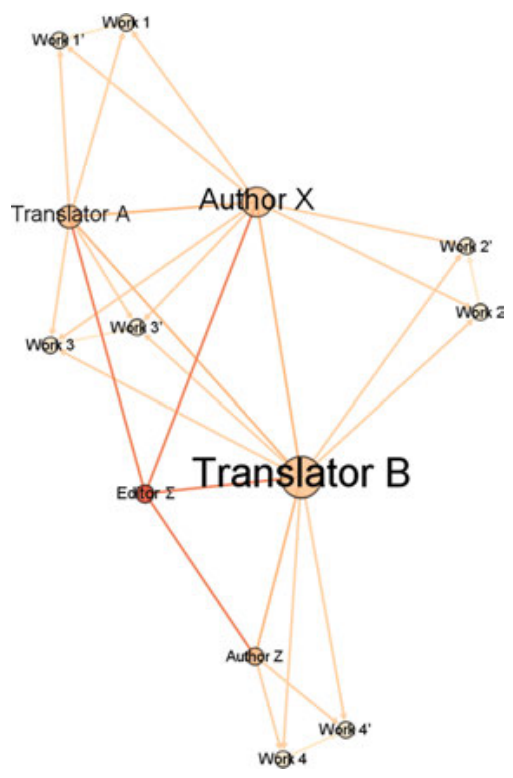

Figura 11

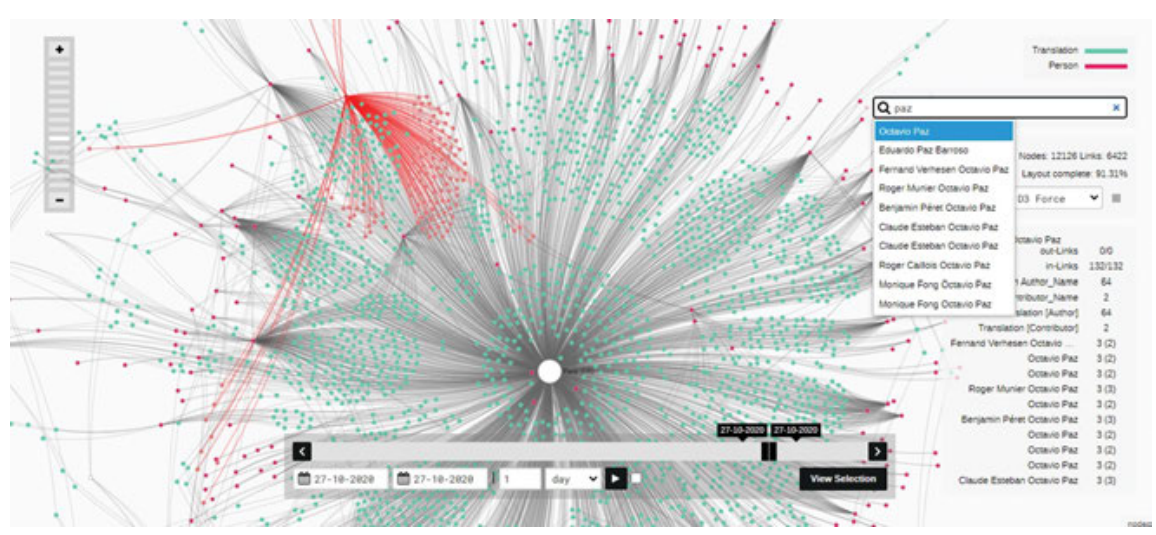

Figura 12 


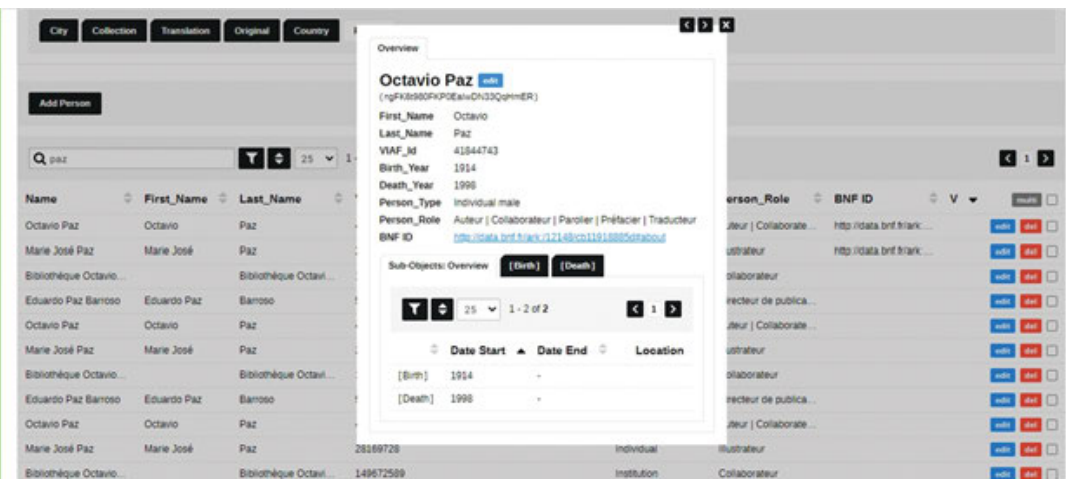

\section{Figura 13}

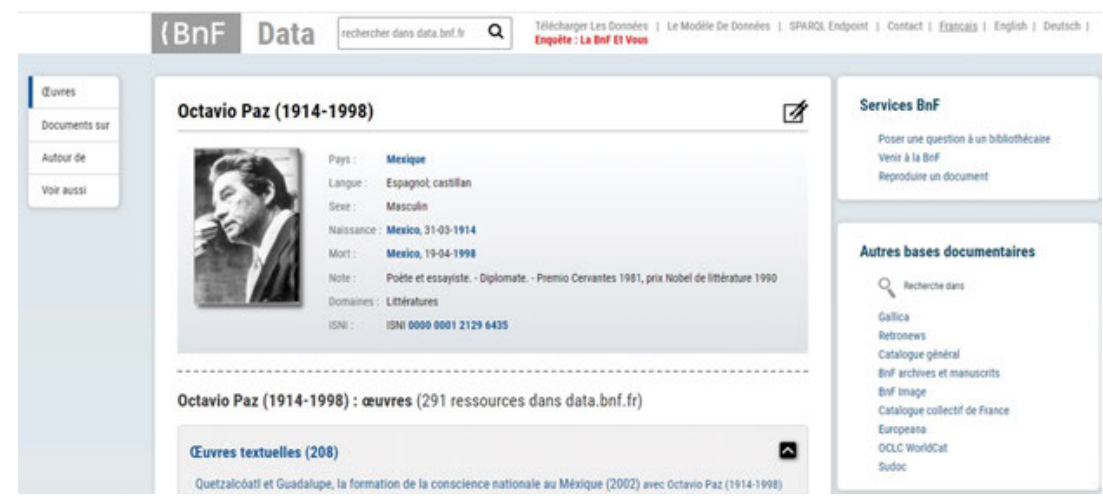

Figura 14

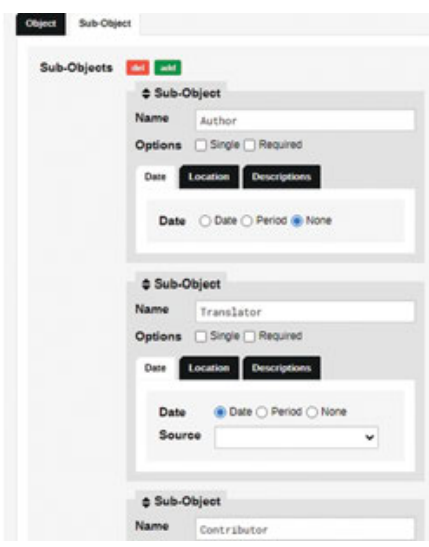

\section{Figura 15}




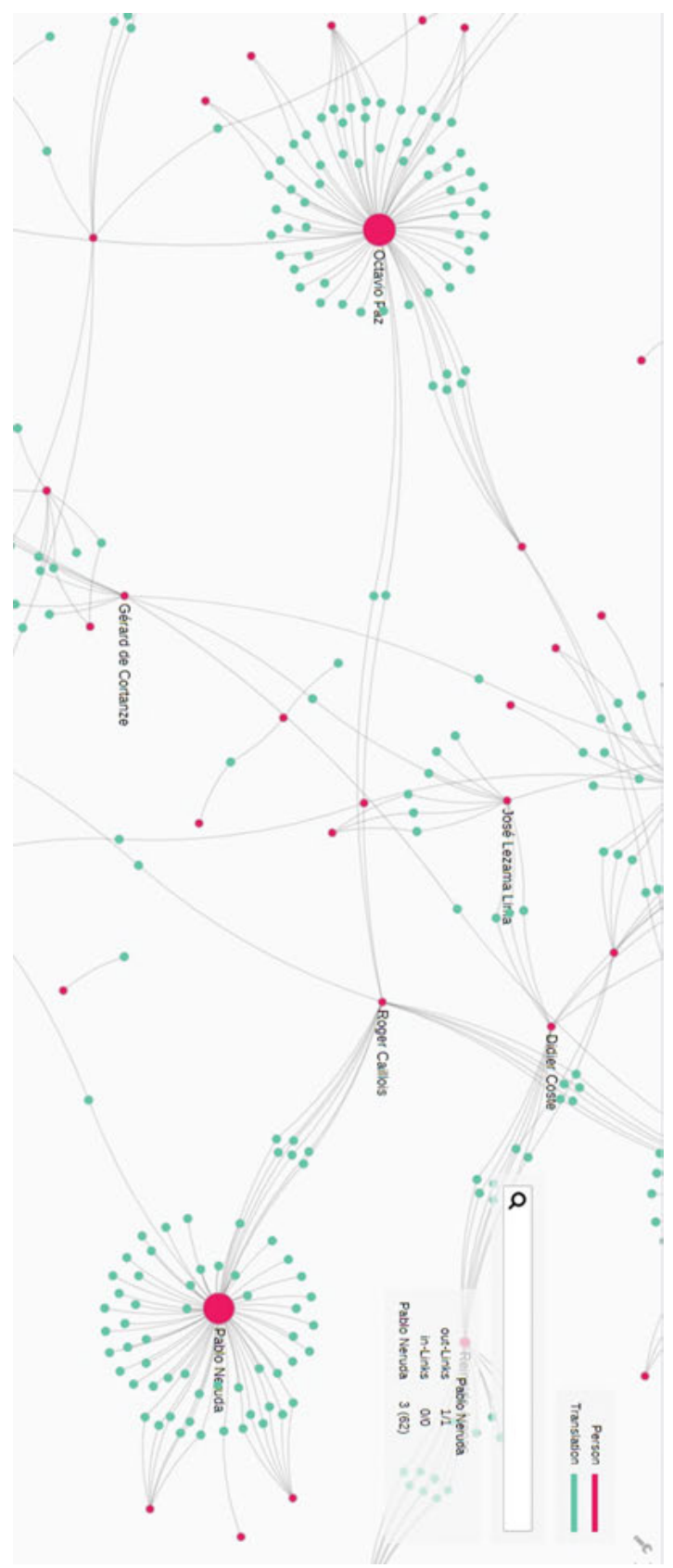

Figura 16 


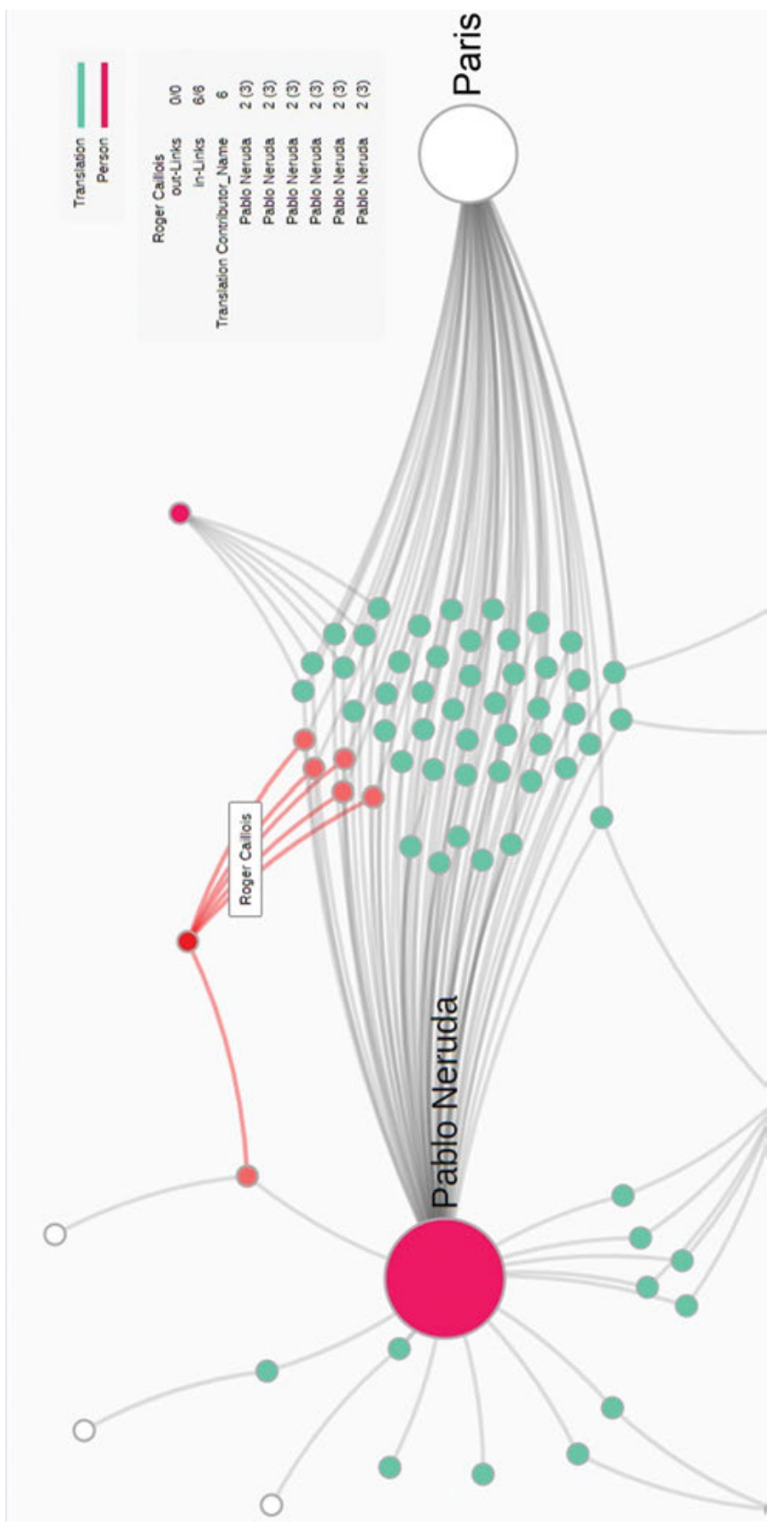

Figura 17 


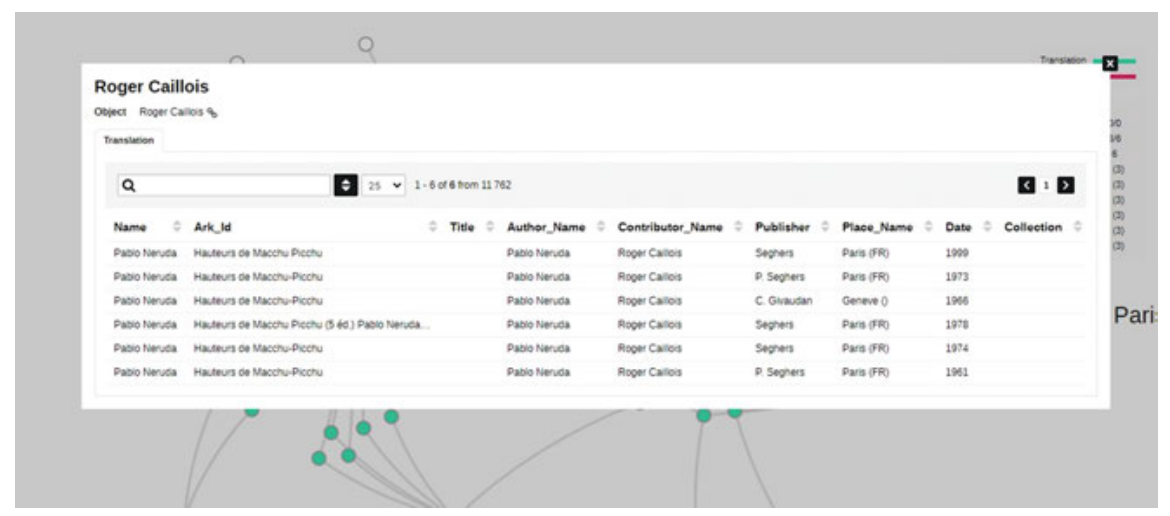

Figura 18 


\section{Bibliografía}

“ACCUEIL | Api.Bnf.Fr” (n.d.). <http://api.bnf.fr/accueil> [última visita: 6/6/2020].

Baillot, Anne (2015): "Visualisation des réseaux: apports, défis et enjeux du travail sur les données historiques". En: Numérisation de masse et traitement des grands corpus de textes utilisant des méthodes des humanités numériques. Stuttgart, Germany. <https://halshs.archives-ouvertes.fr/halshs-01130425>.

- (2016): “La lettre à l'oeuvre. Modèle du réseau, archive du texte”. Thesis. Paris: EHESS. <https://halshs.archives-ouvertes.fr/tel-01562578>.

- (2018): "Reconstruire ce qui manque - ou le déconstruire? Approches numériques des sources historiques”. Digital Humanities Quarterly 012, 1.

Bloomsbury.com (n.d.): "Mexican Literature as World Literature”. Bloomsbury Publishing. <https://www.bloomsbury.com/us/mexican-literature-as-world-literature -9781501374784/> [última visita: 11/4/2021].

Bree, Pim van/Kessels, Geert (2013a): Nodegoat: A Web-Based Data Management, Network Analysis \& Visualisation Environment. LAB1100. <https://nodegoat.net/home,http:// lab1100.com>.

- (2013b): "Towards an Object-Oriented Referencing System: Defining Multiple Forms of Asynchronous Collaboration and Authorship." En: DHLU.

“CubicWeb Semantic Web Framework” (n.d.). <https://www.cubicweb.org/> [última visita: 18/ 6/2020],

Edelstein, Dan (2016): “Interactive Visualization: Letters in Voltaire’s Network”. Mapping the Republic of Letters Data Visualizations. http://republicofletters.stanford.edu.

Edelstein, Dan/Biliana Kassabova (2018): "How England Fell off the Map of Voltaire's Entlightment". En: Modern Intellectual History 17, 1, pp. 1-25. <https://doi.org/10.1017/ S147924431800015X>.

“Entretien avec Albert Bensoussan, réalisé par Gustavo Guerrero, par Ina Salazar et par Stephanie Decante" (n.d). Projet MEDET LAT. <https://www.projet-medetlat.com/entre tiens> [última visita: 17/6/2020].

“EXist-Db - The Open Source Native XML Database” (n.d.). <http://exist-db.org/exist/apps/ho mepage/index.html> [última visita: 6/9/2020].

“Gephi - The Open Graph Viz Platform” (n.d). <https://gephi.org/> [última visita: 21/2/2019].

Grandjean, Martin (2017): “Analisi e Visualizzazioni Delle Reti in Storia. L'esempio Della Cooperazione Intellettuale Della Società Delle Nazioni”. En: Memoria e Ricerca 2, pp. 371-93. <https://doi.org/10.14647/87204>.

- (n.d): “Intellectual Cooperation: Multi-Level Network Analysis of an International Organization”. En: Martin Grandjean (blog). <http://www.martingrandjean.ch/intellec tual-cooperation-multi-level-network-analysis/> [última visita: 23/4/2020].

Guerrero, Gustavo (2018): “La Croix Du Sud (1945-1970): Génesis y Contextos de La Primera Colección Francesa de Literatura Latinoamericana”. En: Re-Mapping World Literature: Writing, Book Markets and Epistemologies between Latin America and the Global South/ Escrituras, Mercados y Epistemologías Entre América Latina y El Sur Global, pp. 199-208. Berlin/Boston: De Gruyter. 〈https://doi.org/10.1515/9783110549577-013>.

- (2020): "Brief History of an Anthology of Mexican Poetry".

Jacomy, Mathieu/Tommaso Venturini/Sebastien Heymann/Mathieu Bastian (2014):

“ForceAtlas2, a Continuous Graph Layout Algorithm for Handy Network Visualization 
Designed for the Gephi Software". En: PLOS ONE 9, 6: e98679. <https://doi.org/10.1371/ journal.pone.0098679>.

Klengel, Susanne (2018): “El Derecho a La Literatura (Mundial y Traducida). Sobre El Sueño Translatológico de La UNESCO". En: Re-Mapping World Literature: Writing, Book Markets and Epistemologies between Latin America and the Global South/Escrituras, Mercados y Epistemologías Entre América Latina y El Sur Global, pp. 131-55. Berlin/Boston: De Gruyter.

Lefebvre, Henri (2009): Dialectical Materialism. Minneapolis: University of Minnesota Press. “Lettres et Textes: Le Berlin Intellectuel Des Années 1800" (n.d.). <https://www.berlinerintellektuelle.eu/?fr> [última visita: 29/5/2020].

“MEDET LAT - YouTube" (n.d.). <https://www.youtube.com/channel/UCXYTIEcjhYK WUiFzjjCqVwA> [última visita: 6/9/2020].

Moretti, Franco (2007): Graphs, Maps, Trees: Abstract Models for a Literary History. London/ New York: Verso.

- (2013): Distant Reading. London: Verso.

Müller, Gesine/Jorge J. Locane/Benjamin Loy (2018): Re-Mapping World Literature: Writing, Book Markets and Epistemologies between Latin America and the Global South/ Escrituras, Mercados y Epistemologías Entre América Latina y El Sur Global. Berlin/ Boston: De Gruyter.

“Nodegoat | About” (n.d.). Nodegoat. <https://nodegoat.net/about> [última visita: 18/6/ 2020].

“Openrefine.Github.Com” (n.d.). <http://openrefine.org/> [última visita: 26/4/2019].

"Programme de Traductions de l'UNESCO, A: Collection UNESCO d'oeuvres Représentatives UNESCO Bibliothèque Numérique” (n.d.). <https://unesdoc.unesco.org/ark:/48223/ pf0000141455_fre.locale=fr> [última visita: 5/6/2020].

“Recogito" (n.d.). <https://recogito.pelagios.org/> [última visita: 6/9/2020].

“Ressource 'Baiser de la femme-araignée / Puig, Manuel”" (n.d.). Mnesys. <https://portailcollections.imec-archives.com/ark:/29414/a011493721979Q7nG1q> [última visita: 5/6/ 2020].

"RStudio | Open Source \& Professional Software for Data Science Teams" (n.d.). <https://rstu dio.com/> [última visita: 17/6/2020].

Schulz, Peter (n.d.): "WP Data Access". WordPress.Org. <https://wordpress.org/plugins/wpdata-access/> [última visita: 3/5/2020].

"Semanticweb_databnf_fr (Data.Bnf.Fr)" (n.d.). <https://data.bnf.fr/fr/semanticweb> [última visita: 4/11/2019].

“Severo-Sarduy" (n.d.). <http://inatheque.ina.fr/doc/TV-RADIO/PH_PHD98013797/severosarduy?rang=1> [última visita: 5/6/2020].

“SPARQL Endpoint de Data.Bnf.Fr | Api.Bnf.Fr” (n.d.). <http://api.bnf.fr/sparql-endpoint-dedatabnffr> [última visita: 6/6/2020].

"VIAF" (n.d.). https://viaf.org/ [última visita: 21/3/2019].

"VIAF (Virtual International Authority File) | OCLC Developer Network" (2018). OCLC. <https:// www.oclc.org/developer/develop/web-services/viaf.en.html> [última visita: 21/3/2019]. 